\title{
On rough $I$ - convergent sequence spaces of fuzzy numbers defined by sequences of Orlicz functions and matrix transformation
}

\author{
Shyamal Debnath ${ }^{1}$, Jayanta Debnath ${ }^{2}$ and Debasish Bhattacharya ${ }^{3}$ \\ ${ }^{1}$ Department of Mathematics,Tripura University, Agartala,India \\ ${ }^{2,3}$ Department of Mathematics, National Institute of Technology, Agartala,India
}

Received: 18 August 2015, Revised: 25 August 2015, Accepted: 3 October 2015

Published online: 18 February 2016.

\begin{abstract}
In this paper we have introduced some classes of new sequence spaces ${ }_{F} c^{I}(r, M, A, p),{ }_{F} c_{0}^{I}(r, M, A, p), F l_{\infty}^{I}(r, M, A, p)$ of fuzzy numbers using the notion of rough $I$-convergence. We study some basic topological and algebraic properties of these spaces. We also investigate some inclusion relations related to these spaces.
\end{abstract}

Keywords: Ideal, rough convergence, Orlicz function, matrix transformation, fuzzy number.

\section{Introduction}

The notion of $I$-convergence was initially introduced by Kostyrko et. al. [7] as a generalization of statistical convergence [4]. Later on it was further investigated from the sequence space point of view and linked with the summability theory by Salat [13], Tripathy and Hazarika [18,19], Tripathy and Mahanta [20], Tripathy and Dutta [17], Tripathy et.al. [23], Savas [15], Savas and Das [14], Kumar [6] and many others. The idea of rough convergence was introduced by Phu [11], who also introduced the concepts of rough limit points and roughness degree. The idea of rough convergence occurs very naturally in numerical analysis and has interesting applications. Aytar [1] extended the idea of rough convergence into rough statistical convergence using the notion of natural density just as usual convergence was extended to statistical convergence. Pal et.al. [10] extended the notion of rough convergence using the concept of ideals which automatically extends the earlier notions of rough convergence and rough statistical convergence. In this paper we have introduced some Orlicz sequence spaces of fuzzy number using the notion of rough $I$ - convergence and studied some algabraic and topological properties of these spaces.

\section{Definitions and preliminaries}

Let $X$ be a non-empty set. Then a family of sets $I \subset 2^{X}$ is said to be an ideal if $I$ is additive, i.e, $A, B \in I \Rightarrow A \cup B \in I$ and hereditary i.e. $A \in I, B \subset A \Rightarrow B \in I$.

A non-empty family of sets $F \subset 2^{X}$ is said to be a filter on $X$ iff 
(i) $\Phi \notin F$

(ii) for all $A, B \in F \Rightarrow A \cap B \in F$

(iii) $A \in F, A \subset B \Rightarrow B \in F$.

An ideal $I \subset 2^{X}$ is called non-trivial if $I \neq 2^{X}$. A non-trivial ideal $I$ is called admissible iff $I \supset\{\{x\}: x \in X\}$. A non-trivial ideal $I$ is maximal if there does not exist any non-trivial ideal $J \neq I$, containing $I$ as a subset. For each ideal $I$ there is a filter $F(I)$ corresponding to $I$ i.e $F(I)=\left\{K \subseteq N: K^{c} \in I\right\}$, where $K^{c}=N-K$. Throughout $w$ denotes the class of all sequences.

Definition 1. A sequence $\left(x_{k}\right) \in w$ is said to be $I$ - convergent to a number $L$ iffor every $\varepsilon>0,\left\{k \in N:\left|x_{k}-L\right|>\varepsilon\right\} \in I$ and we write $I-\lim X_{k}=L$.

Definition 2. A sequence $\left(x_{k}\right) \in w$ is said to be $I$ - null iffor every $\varepsilon>0,\left\{k \in N:\left|x_{k}\right|>\varepsilon\right\} \in I$ and we write $I-$ limx $x_{k}=0$.

Definition 3. A sequence $\left(x_{k}\right) \in w$ is said to be $I$ - bounded if there exists $M>0$ such that $\left\{k \in N:\left|x_{k}\right|>M\right\} \in I$

An Orlicz function $M$ is a continuous, convex, non-decreasing function defined for $x>0$ such that $M(0)=0, M(x)>0$ and $M(x) \rightarrow \infty$ as $x \rightarrow \infty$. If the convexity of Orlicz function is replaced by $M(x+y) \leq M(x)+M(y)$, then this function is called modulus function. An Orlicz function $M$ is said to satisfy $\triangle_{2}$-condition for all values of $u$, if there exists $K>0$ such that $M(2 u) \leq K M(u), u \geq 0$. Let $M$ be an Orlicz function which satisfies $\triangle_{2}$-condition and let $0<\delta<1$. Then for each $t \geq \delta$, we have $M(t)<K \delta^{-1} t M(2)$ for some constant $K>0$. Two Orlicz functions $M_{1}$ and $M_{2}$ are said to be equivalent if there exists positive constants $\alpha, \beta$ and $x_{0}$ such that $\alpha M_{1}(x) \leq M_{2}(x) \leq \beta M_{1}(x)$, for all $0 \leq x<x_{0}$.

Lindenstrauss and Tzafriri [8] studied some Orlicz type sequence spaces defined as follows:

$$
l_{M}=\left\{\left(x_{k}\right) \in w: \sum M\left(\frac{\left|x_{k}\right|}{\rho}\right)<\infty, \text { for } \rho>0\right\}
$$

The space $l_{M}$ with the norm

$$
\|x\|=\inf \left\{\rho>0: \sum M\left(\frac{\left|x_{k}\right|}{\rho}\right) \leq 1\right\},
$$

becomes a Banach space which is called an Orlicz sequence space. The space $l_{M}$ is closely related to $l_{p}$ which is an Orlicz sequence space with $M(t)=|t|^{p}$, for $1 \leq p<\infty$.

Different classes of Orlicz sequence spaces were introduced and studied by Parasar and Choudhury [9], Esi and Et [3], Tripathy and Hazarika [18], Debnath and Debnath [2], Tripathy and Mahanta [21], Tripathy and Sarma [22] and many others.

Let $p=\left(p_{k}\right)$ be any sequence of positive real numbers with $0 \leq p_{k} \leq \sup p_{k}=G$ and $D=\max \left\{1,2^{G-1}\right\}$. Then $\left|a_{k}+b_{k}\right|^{p_{k}} \leq D\left(\left|a_{k}\right|^{p_{k}}+\left|b_{k}\right|^{p_{k}}\right)$ for all $k \in N$ and $a_{k}, b_{k} \in C$. Also $|a|^{p_{k}} \leq \max \left\{1,|a|^{G}\right\}$ for all $a \in C$.

Now we give a brief introduction about the algebra of fuzzy real numbers. Let $D$ be the set of all closed and bounded 
intervals $X=\left[x_{1}, x_{2}\right]$ on the real line $R$. For $X, Y \in D$ we define $X \leq Y$ iff $x_{1} \leq y_{1}$ and $x_{2} \leq y_{2} . d(X, Y)=$ $\max \left\{\left|x_{1}-y_{1}\right|,\left|x_{2}-y_{2}\right|\right\}$ where $X=\left[x_{1}, x_{2}\right]$ and $Y=\left[y_{1}, y_{2}\right]$. Then it can be shown that $(D, d)$ is a complete metric space. Also the relation ' $<$ ' is a partial order relation on $D$. A fuzzy number $X$ is a fuzzy subset of the real line $R$, i.e, a mapping $X: R \rightarrow I=[0,1]$ associating each real number $t$ with its grade of membership $X(t)$. A fuzzy number $X$ is normal if there exists $t_{0} \in R$ such that $X\left(t_{0}\right)=1$. A fuzzy number $X$ is upper semi-continuous if for each $\varepsilon>0, X^{-1}([0, a+\varepsilon))$ is open in the usual topology for all $a \in[0,1)$.

Let $R(I)$ denote the set of all fuzzy numbers which are upper semi-continuous and have a compact support ,i.e, if $X \in R(I)$ then for any $\alpha \in[0,1],[X]^{\alpha}$ is compact where $[X]^{\alpha}=\{t \in R: X(t) \geq \alpha\}$.

The set $R$ of all real numbers can be embedded into $R(I)$ if we define $\bar{r}(t)=\left\{\begin{array}{ll}1 & \text { for } r \neq 0 \\ 0 & \text { for } r=0\end{array}\right.$.

The additive identity and multiplicative identity of $R(I)$ are $\overline{0}$ and $\overline{1}$ respectively. The arithmetic operators on $R(I)$ are defined as follows;

Let $X, Y \in R(I)$ and the $\alpha$-level set $[X]^{\alpha}=\left[X_{1}^{\alpha}, X_{2}^{\alpha}\right]$ and $[Y]^{\alpha}=\left[Y_{1}^{\alpha}, Y_{2}^{\alpha}\right]$ and $\alpha \in[0,1]$. Then we define

$$
\begin{aligned}
& {[X \oplus Y]^{\alpha}=\left[X_{1}^{\alpha}+Y_{1}^{\alpha}, X_{2}^{\alpha}+Y_{2}^{\alpha}\right]} \\
& {[X \ominus Y]^{\alpha}=\left[X_{1}^{\alpha}-Y_{1}^{\alpha}, X_{2}^{\alpha}-Y_{2}^{\alpha}\right]} \\
& {[X \otimes Y]^{\alpha}=\left(\min \left\{X_{i}^{\alpha} Y_{i}^{\alpha}\right\}, \max \left\{X_{i}^{\alpha} Y_{i}^{\alpha}\right\}\right), i=1,2} \\
& {\left[X^{-1}\right]^{\alpha}=\left[\left(X_{2}^{\alpha}\right)^{-1},\left(X_{1}^{\alpha}\right)^{-1}\right], X_{i}^{\alpha}>0 \text { for all } \alpha \in[0,1] .}
\end{aligned}
$$

For $r \in R$ and $X \in R(I)$, the product $r X$ is defined as

$$
r X(t)=\left\{\begin{array}{ll}
X\left(r^{-1} t\right), & \text { for } r \neq 0 \\
0, & \text { for } r=0
\end{array} .\right.
$$

The absolute value $|X|(t)$ is defined by

$$
|X|(t)=\left\{\begin{array}{l}
\max \{X(t), X(-t)\}, \quad \text { for } t>0 \\
0, \quad \text { for } t \leq 0
\end{array} .\right.
$$

Let us define a mapping $\bar{d}: R(I) \times R(I) \longrightarrow R^{+} \bigcup\{0\}$ by $\bar{d}(X, Y)=\operatorname{supd}\left([X]^{\alpha},[Y]^{\alpha}\right)$

It can be shown that $(R(I), \bar{d})$ is a complete metric space.

Definition 4. [22] A sequence of fuzzy numbers $X=\left(X_{k}\right)$ is said to converge to a fuzzy number $X_{0}$ if for every $\varepsilon>0$ there exists a positive integer $n_{0}$ such that $\bar{d}\left(X_{k}, X_{0}\right)<\varepsilon$ for all $n>n_{0}$.

Definition 5. [22] A sequence of fuzzy numbers $X=\left(X_{k}\right)$ is said to be bounded if the set $\left\{X_{k}: k \in N\right\}$ of fuzzy number is bounded. 
Definition 6. [19] A sequence of fuzzy numbers $X=\left(X_{k}\right)$ is said to be $I$-convergent to a fuzzy number $X_{0}$ if for each $\varepsilon>0, A(\varepsilon)=\left\{k \in N: \bar{d}\left(X_{k}, X_{0}\right)>\varepsilon\right\} \in I$.

The fuzzy number $X_{0}$ is called the $I-$ limit of the sequence $\left(X_{k}\right)$ of fuzzy number and we write $I-\lim X_{k}=X_{0}$.

Definition 7. [19] A sequence of fuzzy numbers $X=\left(X_{k}\right)$ is said to be I-bounded if $\exists M>0$ such that $\left\{k \in N: \bar{d}\left(X_{k}, \overline{0}\right)>\right.$ $M\} \in I$.

Let $w^{F}$ denote the collection of all sequences of fuzzy numbers. Then we can give the following definition.

Definition 8. A sequence space $E_{F}$ is said to be solid (or normal) if $\left(Y_{k}\right) \in E_{F}$ whenever $\left(X_{k}\right) \in E_{F}$ and $\left|Y_{k}\right| \leq\left|X_{k}\right|$ for all $k \in N$

Definition 9. A sequence space $E_{F}$ is said to be symmetric if $\left(X_{k}\right) \in E_{F} \Rightarrow\left(X_{\pi(k)}\right) \in E_{F}$ where $\pi$ is a permutation of $N$.

Definition 10. A sequence space $E_{F}$ is said to be monotone if $E_{F}$ contains the canonical pre image of all its step spaces.

Definition 11. [11] A sequence $\left(x_{k}\right)$ is said to be rough convergent (r-convergent) to $x_{0}$ if for every $\varepsilon>0$ there exists a positive integer $n_{0}$ such that $\left\|x_{k}-x_{0}\right\|<r+\varepsilon$ for all $n>n_{0}$, where $r$ is a non negative real number called the convergence degree.

Definition 12. [10] A sequence $\left(x_{k}\right)$ is said to be rough I-convergent to $\left(x_{0}\right)$ if for each $\varepsilon>0,\left\{k \in N:\left\|x_{k}-x_{0}\right\|>r+\varepsilon\right\} \in I$.

Here $x_{0}$ is called the rough I - limit of the sequence $\left(x_{k}\right)$ and we write $I_{r}-$ lim $x_{k}=x_{0}$.

Example 1. (a) Let $I=I_{f}=\{A \subseteq N$ : $A$ is finite $\}$. Then $I_{f}$ is non trivial admissible ideal of $N$ and the corresponding convergence coincides with ordinary convergence.

(b) Let $I=I_{\delta}=\{A \subseteq N: \delta(A)=0\}$ where $\delta(A)$ denotes the asymptotic density of A. Then $I_{\delta}$ is a non-trivial admissible ideal of $\mathrm{N}$ and the corresponding convergence coincide with statistical convergence.

We procure the following well known result:

Lemma 1. A sequence space $E_{F}$ is normal implies that it is monotone.

\section{Some new classes of sequence spaces of fuzzy numbers}

In this section we introduce some new sequence spaces of fuzzy numbers using the notion of rough ideal convergence and also study some properties of these spaces.

Definition 13. A sequence of fuzzy numbers $X=\left(X_{k}\right)$ is said to be rough convergent ( $r$-convergent) to a fuzzy number $X_{0}$ if for every $\varepsilon>0$ there exists a positive integer $n_{0}$ such that $\bar{d}\left(X_{k}, X_{0}\right)<r+\varepsilon$ for all $n>n_{0}$, where $\bar{r}$ is a non-negative real number called the roughness degree.

Definition 14. A sequence of fuzzy numbers $X=\left(X_{k}\right)$ is said to be rough statistical convergent to a fuzzy number $X_{0}$ if for each $\varepsilon>0, \delta\left(\left\{k \in N: \bar{d}\left(X_{k}, X_{0}\right)>r+\varepsilon\right\}\right)=0$. 
Definition 15. A sequence of fuzzy numbers $X=\left(X_{k}\right)$ is said to be rough I-convergent to a fuzzy number $X_{0}$ if for each $\varepsilon>0,\left\{k \in N: \bar{d}\left(X_{k}, X_{0}\right)>r+\varepsilon\right\} \in I$.

The fuzzy number $X_{0}$ is called the rough I - limit of the sequence $\left(X_{k}\right)$ of fuzzy number and we write $I_{r}-\lim X_{k}=X_{0}$.

Let $M=\left(M_{k}\right)$ be a sequence of Orlicz functions, $A=\left(a_{i k}\right)$ be an infinite matrix and $X=\left(X_{k}\right)$ be a sequence of fuzzy numbers. We write $A X=\left(A_{k}(X)\right)$ if $A_{k}(X)=\sum_{k} a_{i k} X_{k}$ converges for each $i$.

We define the following sequence spaces as follows:

${ }_{F} c^{I}(r, M, A, p)=\left\{\left(X_{k}\right) \in w^{F}:\left\{k \in N: \sum_{k=1}^{\infty}\left[M_{k}\left(\frac{\bar{d}\left(A_{k}(X), \bar{L}\right)}{\rho}\right)\right]^{p_{k}} \geq r+\varepsilon\right\} \in I \quad\right.$ for some $\rho>0$ and $\left.\bar{L} \in R(I)\right\}$.

${ }_{F} c_{0}^{I}(r, M, A, p)=\left\{\left(X_{k}\right) \in w^{F}:\left\{k \in N: \sum_{k=1}^{\infty}\left[M_{k}\left(\frac{\bar{d}\left(A_{k}(X), \overline{0}\right)}{\rho}\right)\right]^{p_{k}} \geq r+\varepsilon\right\} \in I \quad\right.$ for some $\rho>0$ and $\left.\bar{L} \in R(I)\right\}$.

${ }_{F} l_{\infty}^{I}(r, M, A, p)=\left\{\left(X_{k}\right) \in w^{F}:\left\{k \in N: \sum_{k=1}^{\infty}\left[M_{k}\left(\frac{\bar{d}\left(A_{k}(X), \bar{L}\right)}{\rho}\right)\right]^{p_{k}} \geq r+K\right\} \in I \quad\right.$ for some $\rho>0, K>0$ and $\left.\bar{L} \in R(I)\right\}$.

${ }_{F} l_{\infty}(M, A, p)=\left\{\left(X_{k}\right) \in w^{F}:\left\{n \in N: \sup _{k} \sum_{k=1}^{\infty}\left[M_{k}\left(\frac{\bar{d}\left(A_{k}(X), \overline{0}\right)}{\rho}\right)\right]^{p_{k}}<\infty\right\}\right\}$.

From definition it is obvious that ${ }_{F} c_{0}^{I}(r, M, A, p) \subset_{F} c^{I}(r, M, A, p) \subset_{F} l_{\infty}^{I}(r, M, A, p) \subset_{F} l_{\infty}(M, A, p)$

Few special cases of the above spaces are as follows.

(i) If $M_{k}(x)=x$ for all $k \in N$, then the above classes of sequences are denoted by ${ }_{F} c^{I}(r, A, p),{ }_{F} c_{0}^{I}(r, A, p),{ }_{F} l_{\infty}^{I}(r, A, p)$ respectively.

(ii) If $p=\left(p_{k}\right)=(1,1,1, \ldots .$.$) then we denote the above spaces by { }_{F} c^{I}(r, M, A),{ }_{F} c_{0}^{I}(r, M, A){ }_{F} l_{\infty}^{I}(r, M, A)$ respectively.

(iii) If we take $A=(C, 1)$, i.e., the Cesaro matrix, then the above classes of sequences are denoted by ${ }_{F} c^{I}(r, w, M, p),{ }_{F} c_{0}^{I}(r, w, M, p){ }_{F} l_{\infty}^{I}(r, w, M, p)$

(iv) If we take $\mathrm{A}=\left(a_{n k}\right)$ is a de la Valeepoussin mean, i.e., $a_{n k}=\left\{\begin{array}{cc}\frac{1}{\lambda_{n}} & \text { for } k \varepsilon I_{n}=\left[n-\lambda_{n}+1, n\right] \\ 0 & \text { otherwise }\end{array}\right.$

where $\left(\lambda_{n}\right)$ is a non-decreasing sequence of positive numbers tending to $\infty$ and $\lambda_{n+1} \leq \lambda_{n}+1, \lambda_{1}=1$, then the above classes of sequences are denoted by ${ }_{F} c_{\lambda}^{I}(r, M, p),{ }_{F} c_{\lambda}^{I}(r, M, p)_{0}, F l_{\lambda}^{I}(r, M, p)_{\infty}$ respectively.

(v) If $r=0$, we get ${ }_{F} c^{I}(M, A, p),{ }_{F} c_{0}^{I}(M, A, p), F l_{\infty}^{I}(M, A, p)$ respectively.

Theorem 1. The spaces ${ }_{F} c^{I}(r, M, A, p),{ }_{F} c_{0}^{I}(r, M, A, p){ }_{F} l_{\infty}^{I}(r, M, A, p)$ are linear space. 
Proof. Let $X=\left(X_{k}\right)$ and $Y=\left(Y_{k}\right)$ be any two elements of ${ }_{F} c^{I}(r, M, A, p)$ and $\alpha, \beta$ be any scalar. Then

$$
\begin{aligned}
& A(\varepsilon)=\left\{k \in N: \sum_{k=1}^{\infty}\left[M_{k}\left(\frac{\bar{d}\left(A_{k}(X), \bar{L}\right)}{\rho}\right)\right]^{p_{k}} \geq r+\varepsilon\right\} \in I . \\
& B(\varepsilon)=\left\{k \in N: \sum_{k=1}^{\infty}\left[M_{k}\left(\frac{\bar{d}\left(A_{k}(Y), \bar{L}\right)}{\rho}\right)\right]^{p_{k}} \geq r+\varepsilon\right\} \in I .
\end{aligned}
$$

By the continuity of the sequence $M=\left(M_{k}\right)$ the following inequality holds:

$$
\begin{aligned}
& \sum_{k=1}^{n}\left[M_{k}\left(\frac{\bar{d}\left(A_{k}(a X+b Y), \overline{0}\right)}{|a| \rho_{1}+|b| \rho_{2}}\right)\right]^{p_{k}} \leq D K \sum_{k=1}^{n}\left[M_{k}\left(\frac{\bar{d}\left(A_{k}(X), \overline{0}\right)}{\rho_{1}}\right)\right]^{p_{k}}+D K \sum_{k=1}^{n}\left[M_{k}\left(\frac{\bar{d}\left(A_{k}(Y), \overline{0}\right)}{\rho_{2}}\right)\right]^{p_{k}} \\
& \leq D \sum_{k=1}^{n}\left[\frac{|a|}{|a| \rho_{1}+|b| \rho_{2}} M_{k}\left(\frac{\bar{d}\left(A_{k}(X), \overline{0}\right)}{\rho_{1}}\right)\right]^{p_{k}}+D \sum_{k=1}^{n}\left[\frac{|b|}{|a| \rho_{1}+|b| \rho_{2}} M_{k}\left(\frac{\bar{d}\left(A_{k}(Y), \overline{0}\right)}{\rho_{1}}\right)\right]^{p_{k}}
\end{aligned}
$$

where $K=\max \left\{1, \frac{|a|}{|a| \rho_{1}+|b| \rho_{2}}, \frac{|b|}{|a| \rho_{1}+|b| \rho_{2}}\right\}$.

From the above relation we get the following:

$$
\begin{aligned}
& \left\{k \in N: \sum_{k=1}^{\infty}\left[M_{k}\left(\frac{\bar{d}\left(A_{k}(a X+b Y), \overline{0}\right)}{|a| \rho_{1}+|b| \rho_{2}}\right)\right]^{p_{k}} \geq r+\frac{\varepsilon}{2}\right\} \subseteq \\
& \left\{k \in N: \sum_{k=1}^{\infty} D K\left[M_{k}\left(\frac{\bar{d}\left(A_{k}(X), \overline{0}\right)}{\rho_{1}}\right)\right]^{p_{k}} \geq r+\frac{\varepsilon}{2}\right\} \\
& \bigcup\left\{k \in N: \sum_{k=1}^{\infty} D K\left[M_{k}\left(\frac{\bar{d}(A(Y), \overline{0})}{\rho_{2}}\right)\right]^{p_{k}} \geq r+\frac{\varepsilon}{2}\right\}
\end{aligned}
$$

This completes the proof.

Theorem 2. The space ${ }_{F} c^{I}(r, M, A, p),{ }_{F} c_{0}^{I}(r, M, A, p),{ }_{F} l_{\infty}^{I}(r, M, A, p)$ are paranormed spaces (not totally paranormed) with the paranorm $g$ defined by:

$g(x)=\inf \left\{\rho^{\frac{p_{k}}{H}}: \sup _{k} M_{k}\left(\frac{\bar{d}\left(A_{k}(X), \overline{0}\right)}{\rho}\right) \leq r\right.$, for $\left.\rho>0\right\}$, where $H=\max \left\{r, \sup _{k} p_{k}\right\}$.

Proof. We prove the result for ${ }_{F}^{I}(r, M, A, p)$ only. The others can be proved similarly.

It is obvious that $g(0)=0, g(-x)=g(x)$ and it can be easily shown that $g(x+y) \leq g(x)+g(y)$.

Let $t_{n} \rightarrow t$, where $t_{n}, t \in R(I)$ and let $g\left(x_{n}-x\right) \rightarrow 0$, as $n \rightarrow \infty$. To prove that $g\left(t_{n} x_{n}-t x\right) \rightarrow 0$, as $n \rightarrow \infty$. We put

$$
A=\left\{\rho_{1}>0: \sup _{k}\left[M_{k}\left(\frac{\bar{d}\left(A_{k}(X), \overline{0}\right)}{\rho_{1}}\right)\right]^{p_{k}} \leq r\right\}
$$

and

$$
B=\left\{\rho_{2}>0: \sup _{k}\left[M_{k}\left(\frac{\bar{d}\left(A_{k}(x), \overline{0}\right)}{\rho_{2}}\right)\right]^{p_{k}} \leq r\right\}
$$

By the continuity of the sequence $M=\left(M_{k}\right)$ we observe that 


$$
\begin{aligned}
& M_{k}\left(\frac{\bar{d}\left(A_{k}\left(t_{n} X_{n}-t X\right), \overline{0}\right)}{\left|t_{n}-t\right| \rho_{1}+|t| \rho_{2}}\right) \leq M_{k}\left(\frac{\bar{d}\left(A_{k}\left(t_{n} X_{n}-t X_{n}\right), \overline{0}\right)}{\left|t_{n}-t\right| \rho_{1}+|t| \rho_{2}}\right)+M_{k}\left(\frac{\bar{d}\left(A_{k}\left(t X_{n}-t X\right), \overline{0}\right)}{\left|t_{n}-t\right| \rho_{1}+|t| \rho_{2}}\right) \\
& \leq \frac{\left|t_{n}-t\right| \rho_{1}}{\left|t_{n}-t\right| \rho_{1}+|t| \rho_{2}} M_{k}\left(\frac{\bar{d}\left(A_{k}\left(X_{n}\right), \overline{0}\right)}{\rho_{1}}\right)+\frac{|t| \rho_{2}}{\left|t_{n}-t\right| \rho_{1}+|t| \rho_{2}} M_{k}\left(\frac{\bar{d}\left(A_{k}\left(X_{n}-X\right), \overline{0}\right)}{\rho_{2}}\right)
\end{aligned}
$$

From the above inequality it follows that

$$
\sup _{k}\left[M_{k}\left(\frac{\bar{d}\left(A_{k}\left(t_{n} X_{n}-t X\right), \overline{0}\right)}{\left|t_{n}-t\right| \rho_{1}+|t| \rho_{2}}\right)\right]^{p_{k}} \leq r
$$

and hence

$$
\begin{aligned}
& g\left(t_{n} X_{n}-t X\right)=\inf \left\{\left(\left|t_{n}-t\right| \rho_{1}+|t| \rho_{2}\right)^{\frac{p_{k}}{H}}: \rho_{1} \in A, \rho_{2} \in B\right\} \\
& \leq\left(\left|t_{n}-t\right|\right)^{\frac{p_{k}}{H}} \inf \left\{\rho_{1}^{\frac{p_{k}}{H}}: \rho_{1} \in A\right\}+(|t|)^{\frac{p_{k}}{H}} \inf \left\{\rho_{2}^{\frac{p_{k}}{H}}: \rho_{2} \in B\right\} \\
& \leq \max \left\{\left|t_{n}-t\right|,\left(\left|t_{n}-t\right|\right)^{\frac{p_{k}}{H}}\right\} g\left(X_{n}\right)+\max \left\{|t|,(|t|)^{\frac{p_{k}}{H}}\right\} g\left(X_{n}-X\right) .
\end{aligned}
$$

As $g\left(X_{n}\right) \leq g(X)+g\left(X_{n}-X\right)$ for all $n \in N$, hence our assumption the right hand side of the above relation tends to zero as $n \rightarrow \infty$. This completes the proof.

Proposition 1. Let $M=\left(M_{k}\right)$ and $N=\left(N_{k}\right)$ be sequences of Orlicz functions. Then the following hold:

(i) ${ }_{F} c_{0}^{I}(N, A, p) \subseteq_{F} c_{0}^{I}(M o N, A, p)$, provided $p=\left(p_{k}\right)$ such that $G_{0}=\inf p_{k}>0$.

(ii) ${ }_{F} c_{0}^{I}(M, A, p) \bigcap_{F} c_{0}^{I}(N, A, p) \subseteq_{F} c_{0}^{I}(M+N, A, p)$

Theorem 3. The spaces ${ }_{F} c^{I}(r, M, A, p),{ }_{F} c_{0}^{I}(r, M, A, p)$ are normal and monotone.

Proof. Let $X=\left(X_{k}\right) \in c_{0}^{I}(r, M, A, p)$ and $Y=\left(Y_{k}\right)$ be such that $\bar{d}\left(Y_{k}, \overline{0}\right) \leq \bar{d}\left(X_{k}, \overline{0}\right)$. Then for $\varepsilon>0$,

$$
\begin{gathered}
\left\{k \in N: \sum_{k=1}^{\infty}\left[M_{k}\left(\frac{\bar{d}(A(X), \overline{0})}{\rho}\right)\right]^{p_{k}} \geq r+\varepsilon\right\} \\
\supseteq\left\{k \in N: \sum_{k=1}^{\infty}\left[M_{k}\left(\frac{\bar{d}\left(A_{k}(Y), \overline{0}\right)}{\rho}\right)\right]^{p_{k}} \geq r+\varepsilon\right\} \in I
\end{gathered}
$$

Then the result follows from the above relation. Thus the space ${ }_{F} c_{0}^{I}(r, M, A, p)$ is normal and hence monotone by lemma 2.14. Similarly for the other.

Proposition 2. If $I$ is neither maximal nor $I=I_{f}$ then the spaces ${ }_{F} c^{I}(r, M, A, p),{ }_{F} c_{0}^{I}(r, M, A, p)$ are not symmetric in general.

Theorem 4. The spaces ${ }_{F} c^{I}(r, M, A, p),{ }_{F} c_{0}^{I}(r, M, A, p)$ are sequence algebra. 
Let $X=\left(X_{k}\right)$ and $Y=\left(Y_{k}\right)$ be any element of ${ }_{F} c^{I}(r, M, A, p)$. Let $\varepsilon>0$ be given. Then

$$
\begin{aligned}
& A(\varepsilon)=\left\{k \in N: \sum_{k=1}^{\infty}\left[M_{k}\left(\frac{\bar{d}\left(A_{k}(X), \bar{L}\right)}{\rho}\right)\right]^{p_{k}} \geq r+\varepsilon\right\} \in I . \\
& B(\varepsilon)=\left\{k \in N: \sum_{k=1}^{\infty}\left[M_{k}\left(\frac{\bar{d}\left(A_{k}(Y), \bar{L}\right)}{\rho}\right)\right]^{p_{k}} \geq r+\varepsilon\right\} \in I . \\
& C(\varepsilon)=\left\{k \in N: \sum_{k=1}^{\infty}\left[M_{k}\left(\frac{\bar{d}\left(A_{k}(X \otimes Y), \bar{L}\right)}{\rho}\right)\right]^{p_{k}} \geq r+\varepsilon\right\} \in I .
\end{aligned}
$$

Now it can be easily shown that $C(\varepsilon) \subset A\left(\varepsilon_{1}\right) \cup B\left(\varepsilon_{2}\right)$ and $C(\varepsilon) \in{ }_{F} c^{I}(r, M, A, p)$.

Theorem 5. The spaces ${ }_{F} c^{I}(r, M, A, p),{ }_{F} c_{0}^{I}(r, M, A, p)$ are not convergence free in general.

Proof. Proof is easy, so omitted.

\section{References}

[1] S. Aytar, Rough Statistical Convergence, Numer. Funct. Anal. Optimiz., 29(3) (2008), 291-303.

[2] S.Debnath and J. Debnath, On I-statistically convergent sequence spaces defined by sequences of Orlicz functions using matrix transformation, Proyec. J. Math, 33(3) (2014), 277-285.

[3] A.Esi and M.Et., Some new spaces defined by Orlicz functions, Indian J. Pure. Appl. Math., 31(8)(2000), 967-972.

[4] H.Fast, Sur la convergence statistique, Colloq. Math., 2(1951), 241-244.

[5] J.A.Fridy, On statistical convergence, Analysis , 5(1985), 301-313.

[6] V.Kumar and K.Kumar, On the ideal convergence of sequences of fuzzy numbers, Inform. Sci., 178 (2008), 4670-4678.

[7] P.Kostyrko, T.Salat and W.Wilczynski, I-convergence, Real Anal.Exchange, 26(2)(2000/2001), 669-686.

[8] J.Lindenstrauss and L.Tzafriri, On Orlicz sequence spaces, Israel J. Math, 101(1971), 379-390.

[9] S.D.Parashar, B.Choudhury, Sequence space defined by Orlicz functions, Indian J. Pure. Appl. Math., 25(14)(1994), 419-428.

[10] S. K. Pal, D. Chandra and S. Dutta, Rough ideal convergence, Hacee. J. Math. Stat., 42(6)(2013), 633- 640.

[11] H. X. Phu, Rough convergence in normed linear spaces, Numer. Funct. Anal. Optimiz., 22(2001), 201-224.

[12] H. X.Phu, Rough convergence in infinite dimensional normed spaces, Numer. Funct. Anal. Optimiz., 24(2003), $285-301$.

[13] T.Salat, On statistically convergent sequences of real numbers, Math. Slovaca., 30(1980),139-150.

[14] E.Savas and P.Das, A generalized statistical convergence via ideals, Appl. Math. Letters , 24(2011),826-830.

[15] E.Savas, Some I-convergent sequence spaces of fuzzy numbers defined by infinite matrix, Math. Compt. Appl., 18(2)(2013), 84-93.

[16] I. J.Schoenburg, The integrability of certain functions and related summability methods, American Math. Mon., 66(1951),361-375.

[17] B.C.Tripathy and A.J.Dutta, On I-acccleration convergence of sequences of fuzzy real numbers, Math. Modell. Analysis, 17(4) (2012), 549-557.

[18] B.C.Tripathy and B.Hazarika, Some I-convergent sequence spaces defined by orlicz functions, Acta Math. Appl. Sin. , 27(1)(2011),149-154.

[19] B.C.Tripathy and B.Hazarika, Paranorm I-convergent sequence spaces, Math. Slovaca 59(4) (2009), 485-494.

[20] B.C.Tripathy and S.Mahanta, On I-acccleration convergence of sequences, Jour. Franklin Inst., 347(2010), 591-598.

[21] B.C.Tripathy and S.Mahanta, On a class of generalized lacunary difference sequence spaces defined by Orlicz function, Acta Math. Applicata Sinica (Eng. Ser.), 20(2)(2004) 231-238. 
[22] B.C.Tripathy and B.Sarma, Sequence Spaces of fuzzy real numbers defined by Orlicz functions, Math. Slovaca, 58(5) (2008), 621-628

[23] B.C.Tripathy, M.Sen and S. Nath, I-convergence in probabilistic n-normed spaces, Soft Comput., 16(2012), 1021-1027. 